\title{
3-Dimensional computed tomography imaging of the ring-sling complex with non-operative survival case in a 10-year-old female
}

\author{
HIRONOBU FUKUDA ${ }^{1}$, GEORGE IMATAKA ${ }^{1}$, FABRIZIO DRAGO ${ }^{2}$, \\ KOSAKU MAEDA $^{3}$ and SHIGEMI YOSHIHARA ${ }^{1}$ \\ ${ }^{1}$ Department of Pediatrics, Dokkyo Medical University, Tochigi, Japan; ${ }^{2}$ Department of Pediatric Cardiology \\ and Cardiac Surgery, Pediatric Cardiology and Cardiac Arrhythmia Complex Unit, \\ Bambino Gesù Children's Hospital and Research Institute, Rome, Italy; \\ ${ }^{3}$ Department of Pediatric Surgery, Kobe Children's Hospital, Kobe, Japan
}

Received May 1, 2017; Accepted July 5, 2017

DOI: $10.3892 /$ etm.2017.4821

\begin{abstract}
We report a case of a 10-year-old female patient who survived ring-sling complex without surgery. The patient had congenital wheezing from the neonatal period and was treated after a tentative diagnosis of infantile asthma. The patient suffered from allergy and was hospitalized several times due to severe wheezing, and when she was 22 months old, she was diagnosed with ring-sling complex. We used a segmental $4 \mathrm{~mm}$ internal diameter of the trachea for 3-dimensional computed tomography (3D-CT). Bronchial asthma is considered an exacerbating factor in infantile period and frequently required treatment with bronchodilator. After the age of 10 , the patient had recurrent breathing difficulties during physical activity and during night time, and this condition was assessed to be related to the pressure from the blood vessel on the ring. We repeated the 3D-CT evaluation later and discovered that the internal diameter of the trachea had grown to $5 \mathrm{~mm}$. Eventually, patient's breathing difficulties disappeared after the treatment of bronchial asthma and restriction of physical activities. Our patient remained in stable condition without undergoing any surgical procedures even after she passed the age of 10 .
\end{abstract}

\section{Introduction}

One of the main causes of wheezing and respiratory distress in newborns and infants is pulmonary sling (1). Pulmonary sling is a congenital bronchial malformation in which the left pulmonary artery originates from the right pulmonary artery and its artery passes between the trachea and the esophagus

Correspondence to: Dr George Imataka, Department of Pediatrics, Dokkyo Medical University, 880 Kitakobayashi, Mibu, Shimotsuga, Tochigi 321-0293, Japan

E-mail: geo@dokkyomed.ac.jp

Key words: 3D-CT, pulmonary sling, lung agenesis, tracheal stenosis, vascular compression and leads to the left hilar (2,3). Pulmonary sling may be treated by cardiovascular surgery, and this treatment allows us to release the pressure of the trachea by reconstructing surgery on the left pulmonary artery (4). The ring-sling complex is a rare malformation, which complicates the congenital tracheal stenosis caused by pulmonary sling due to the circumferential cricoid of the trachea. The prognosis for this condition is relatively poor and this may be explained by the fact that tracheal stenosis is not lifted, even after surgery on the left pulmonary artery (4). We studied a 22-month-old patient diagnosed with ring-sling complex triggered by recurrent wheezing after birth. The patient survived until the age of 10 without undergoing any surgical procedures. In this report, we explain the patient's clinical course using the results obtained from our examinations with 3-dimensional computed tomography (3D-CT) imaging.

\section{Case report}

The patient was born with an emergency caesarean section with a declining fetal heart rate at 41 weeks gestation. She weighed 2,860 $\mathrm{g}$ at birth with no external congenital abnormality. Although birth asphyxia was not noted, the patient had repeated wheezing during the neonatal period. When she was just 3 months old, the patient was hospitalized in another hospital and was diagnosed with croup syndrome caused by a viral infection. At 6 months old, she was hospitalized again, and this time she was diagnosed with a bronchopneumonia accompanying with $\mathrm{RS}$ virus infection. At that time, she was tentatively diagnosed with infantile asthma, and subsequently she was hospitalized in different occasions because of severe wheezing and breathing difficulties.

At 22 months, she was admitted to Dokkyo Medical University due to bronchial asthma and dyspnea. During the examination, in chest auscultation, wheezing was heard during both inhalation and exhalation, therefore she was hospitalized for a more detailed examination. Allergy tests showed positive RAST reaction to egg antigen, while no abnormality in laryngeal fiber was detected during the examinations conducted in our otolaryngology department. Cardiac ultrasound revealed no noticeable cardiac structural abnormality, however, chest 


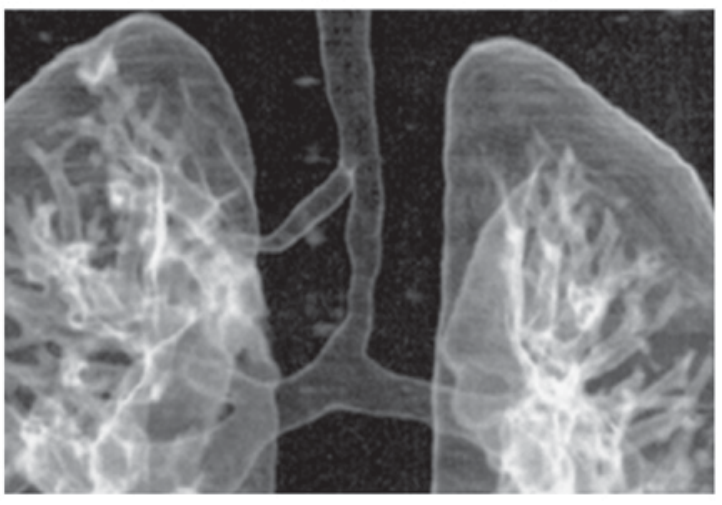

Figure 1. At 1 year and 10 months of 3D-CT, trachea-bronchi and congenital tracheal stenosis was observed. Minimum bronchial inner diameter of the tracheal stenosis is $4 \mathrm{~mm}$ and type of tracheal stanosis was segmental type. 3D-CT, 3-dimensional computed tomography.

X-ray showed an enhancement of both side vascular shadows. Also, the ascending aorta detected on the right side of the trachea and the bronchus was displaced to the left side. Results obtained from contrast 3D-CT examinations, showed some interesting findings of circumflex aorta and tracheal bronchus and the patient was diagnosed as 'ring-sling complex'. At that time, the inner diameter of the most constricted part of the main trachea was $4 \mathrm{~mm}$ (Fig. 1). After she was treated with oxygen, bronchodilator and steroid, her respiratory condition improved significantly. We studied the indication of the vascular surgery, however our policy was to continue observing her growth, her respiration and circulation conditions.

When she was 10 , the patient started showing breathing complications. She was aware of the fact that her dyspnea was different from the asthma symptoms. Breathing complications appeared during the physical activity, and improved when the activity was halted and she rested for at least $15 \mathrm{~min}$. Moreover, she also suffered from recurrent nocturnal dyspnea, with chest auscultation during the night and this condition was accompanied with inspiratory wheezing. She was hospitalized when these problems occurred more frequently for more than 2 months. Chest X-ray showed enhanced bilateral vascular shadow accompanied with an increase in pulmonary blood flow (Fig. 2A). The ascending aorta was located on the right side of the trachea and the bronchus was displaced to the left side, while the thymus was physiologically enlarged at puberty. Chest 3D-CT showed a tracheal bronchus, anomalous origin of the left pulmonary artery, pulmonary artery sling, right aortic arch, right inferior vena cava remnant, and mild tracheal stenosis. Chest contrast 3D-CT also revealed narrowing of the tracheal lumen where the left pulmonary artery crossed behind the trachea. However, CT did not reveal any obvious exacerbation of tracheal stenosis, and the minor axis was $5 \mathrm{~mm}$ (Figs. 2B and C, and 3). Her bronchial asthma stabilized with medication.

The cause of dyspnea was diagnosed to be linked to blood vessel pressure caused by the vascular rings triggered by conditions such as bronchial asthma, common cold, exercise and night time sleep. As a result, we initially considered surgical intervention, but interestingly, her breathing problems disappeared after the treatment of bronchial asthma and a reduction in physical activities. Meanwhile, we carefully and thoroughly, monitored the patient's breathing and circulation conditions.

\section{Discussion}

Ring-sling complex is a rare but potentially life-threatening disorder that often leads to severe respiratory insufficiency, particularly in neonates and infants $(5,6)$. Although it has been reported that symptoms can be alleviated without surgery $(6,7)$, surgical intervention is often required in symptomatic patients $(4,5,7)$. As a pre-evaluation step before reconstructive surgery on trachea malformation and stenosis, 3D-CT examination is considered to be a valuable tool. Kagadis et al (4) wrote about the usefulness of 3D-CT for congenital tracheal stenosis and tracheal malformations. The clinical course in our case seemed to have dyspnetic symptom due to the pressure of the main trachea by the vascular ring from the neonatal period. 3D-CT showed that our patient had a tracheal inner diameter of $4 \mathrm{~mm}$ at 22 months old. Tracheal stenosis of pulmonary sling usually ranges from segmental type to generalized type, and luckily our case had a segmental type. Her bronchial asthma was an exacerbation factor, but by managing the bronchial asthma, she grew past the infancy period. Problematic breathing outbreaks were regularly observed after the age of 10 , however, 3D-CT showed that her internal trachea diameter was grown to over $5 \mathrm{~mm}$. Typically, ring-sling complex cause wheezing throughout the early stages
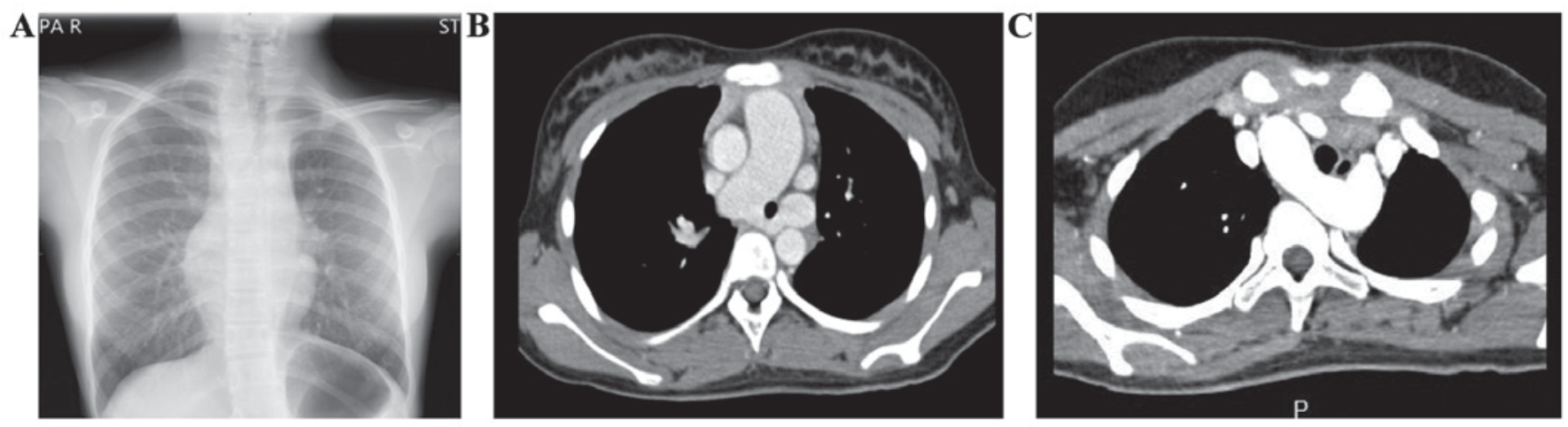

Figure 2. (A) In the chest X-ray at the age of 10, the bronchi is deviated to the left side and hypertrophy of the thorax is observed. (B and C) The right aortic arch where the ascending aorta is located on the right side of the trachea and the pulmonary artery sling heading toward the hilar side through the posterior side of the trachea. 


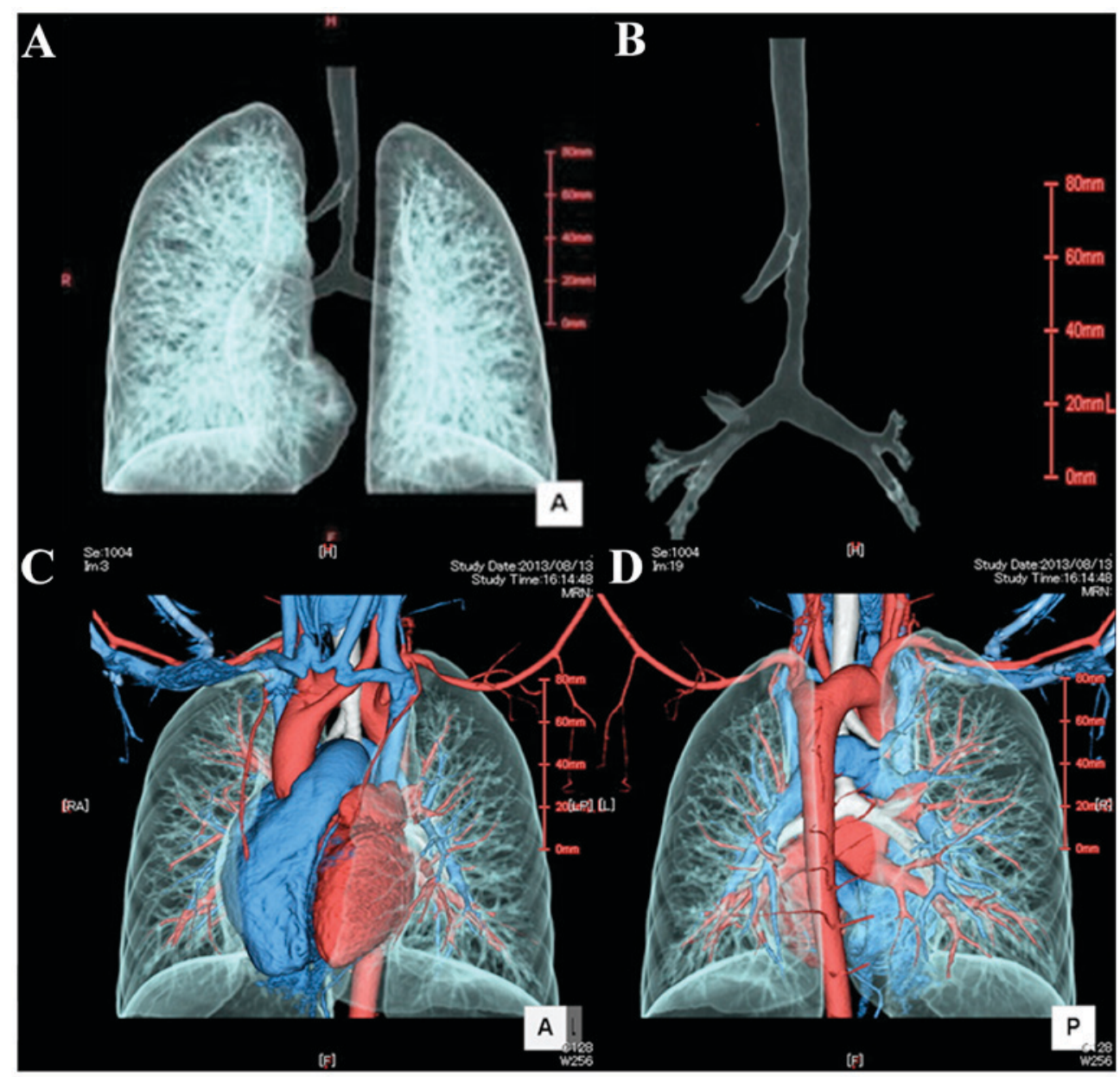

Figure 3. (A and B) There is a bronchus diverging from the right main bronchus and heading towards the upper right lobe. The minimum inner diameter of the tracheal constriction of the segment is $5 \mathrm{~mm}$. (C and D) A right aorta with an abnormality in the origin of the left subclavian artery is observed. The right inferior vena cava remnant is recognized.

of infancy, which can be fatal in the case of young infants $(8,9)$. There are other studies reporting that some patients may reach adulthood without any surgical intervention while their conditions are monitored closely (10). There are some ring-sling complex cases in which the prognosis does not improve by surgery, and there a small group that can manage to survive without any surgical intervention.

We will continue our monitoring and evaluating the condition of trachea and surrounding blood vessels, and, of course, we would consider surgical intervention should respiratory problems intensify. We concluded that in the case of congenital wheezing under diagnosis of ring-sling complex during early infantile stage, it is necessary to carefully determine the necessity of surgical intervention by evaluating the inner diameter of the trachea by congenital tracheal stenosis using radiological technique with $3 \mathrm{D}-\mathrm{CT}$.

\section{References}

1. Kir M, Saylam GS, Karadas U, Yilmaz N, Çakmakçi H, Uzuner N, Güzeloğlu M, Ugurlu B and Oto Ö: Vascular rings: Presentation, imaging strategies, treatment, and outcome. Pediatr Cardiol 33: 607-617, 2012.

2. Hraska V, Photiadis J, Haun C, Schindler E, Schneider M, Murin P and Asfour B: Pulmonary artery sling with tracheal stenosis. Multimed Man Cardiothorac Surg 2009: mmcts.2008.003343, 2009 .
3. Son JS, Ryu JW and Chang YP: Multidetector computed tomography of the ring-sling complex in an infant. Pediatr Cardiol 30: 210-211, 2009.

4. Kagadis GC, Panagiotopoulou EC, Priftis KN, Vaos G, Nikiforidis GC and Anthracopoulos MB: Preoperative evaluation of the trachea in a child with pulmonary artery sling using 3-dimensional computed tomographic imaging and virtual bronchoscopy. J Pediatr Surg 42: E9-E13, 2007.

5. Hofferberth SC, Watters K, Rahbar R and Fynn-Thompson F: Management of congenital tracheal stenosis. Pediatrics 136: e660-e669, 2015.

6. Antón-Pacheco JL, Cano I, Comas J, Galletti L, Polo L, García A, López M and Cabezalí D: Management of congenital tracheal stenosis in infancy. Eur J Cardiothorac Surg 29: 991-996, 2006.

7. Cheng W, Manson DE, Forte V, Ein SH, MacLusky I, Papsin BC Hechter S and Kim PC: The role of conservative management in congenital tracheal stenosis: An evidence-based long-term follow-up study. J Pediatr Surg 41: 1203-1207, 2006.

8. Horak E,Hammerer I, Schmitzberger R and Gassner I: Pulmonary sling or ring-sling complex? respiratory problems - diagnosis, therapy, prognosis. Klin Padiatr 209: 121-126, 1997 (In German).

9. Yo CH, Lee CC, Chang CI, Chen SJ, Wu MH and Lu FL: Ring-sling complex: Report of one case. Acta Paediatr Taiwan 46: 311-313, 2005.

10. Lee JC, Kim GS, Lee SJ, Yoo CG, Kim YW, Han SK and Shim YS: An adult case of pulmonary sling with complete tracheal ring. Korean J Intern Med 11: 175-177, 1996. 